Michael Blauberger/Tilman Krüger/Susanne K. Schmidt

\title{
Die Pfadabhängigkeit internationaler Verrechtlichung
}

EU und WTO im Vergleich

\begin{abstract}
Dieser Beitrag liefert eine historisch-institutionalistische Erklärung für die Frage, weshalb und unter welchen Bedingungen internationale Gerichte den Prozess der internationalen Verrechtlichung auch gegen staatlichen Widerstand vorantreiben. Ausgehend von der Interpretation der Warenverkehrsfreiheit durch den Europäischen Gerichtshof (EuGH) wird gezeigt, wie einmal vollzogene Schritte der Internationalisierung durch pfadabhängige Rechtsprechung auf andere Bereiche übertragen werden, obwohl die funktionalen Bedürfnisse dort teilweise andere sind. Zwei Vergleichsfälle - unionsintern zum freien Kapitalverkehr und extern zur Welthandelsorganisation (WTO) - untersuchen die Voraussetzungen und Grenzen dieser internationalen Verrechtlichung durch pfadabhängige Rechtsprechung. Ein großes Fallvolumen ist nötig, damit die juristische Präzedenzlogik ihre eigene Wirkmacht entwickelt und es bedarf privater und supranationaler Akteure, die das Fallrecht aufgreifen und dadurch positiv verstärken.
\end{abstract}

\section{Einleitung}

Prozesse internationaler Verrechtlichung haben die ehemals enge Verbindung von (nationalstaatlichem) Recht und Territorium gelockert. ${ }^{1}$ Das entstehende Mehrebenensystem überlappender Rechtsordnungen und die Dichte an internationalem und supranationalem Recht betreffen auch vormals exklusiv nationale Regelungsbereiche. Entsprechend ist das Interesse an Verrechtlichungsprozessen jenseits des Staates nicht nur in der Rechtswissenschaft, sondern auch in den politikwissenschaftlichen Bereichen der Internationalen Beziehungen (IB) und der Europaforschung gestiegen (Abbott et al. 2000; List/Zangl 2003). Insbesondere die Rolle supranationaler Gerichte und gerichtsähnlicher Streitbeilegungsinstanzen erfährt zunehmende Aufmerksamkeit (Alter 2008; Zangl 2009a). Die Rechtsprechung entfaltet dabei nicht nur für die Regeleinhaltung (compliance, vgl. Zürn 2005), sondern auch für grundlegende Verfassungsfragen Bedeutung, etwa wenn sie über die Abgrenzung überlappender Rechtsordnungen zu entscheiden hat.

In diesem Kontext bieten wir eine historisch-institutionalistische Erklärung für die Frage, weshalb und unter welchen Bedingungen internationale Gerichte die in-

1 Wir danken den anonymen GutachterInnen der ZIB und Klaus Dingwerth für ihre ausführlichen und sehr hilfreichen Kommentare sowie der Deutschen Forschungsgemeinschaft für die großzügige Unterstützung im Rahmen des Bremer Sonderforschungsbereiches 597 »Staatlichkeit im Wandel«. In der Ausarbeitung des Pfadabhängigkeitsarguments haben wir sehr von Fritz Scharpfs Kommentaren profitiert. 
ternationale Verrechtlichung auch gegen staatlichen Widerstand vorantreiben. Wir zeigen, dass internationale Rechtsprechung eine Eigendynamik entwickeln kann, die pfadabhängig auf weitere Internationalisierung ausgerichtet ist. Ausgehend von der Rechtsprechung des EuGH zum freien Warenverkehr in der Europäischen Union (EU) wird gezeigt, wie einmal vollzogene Schritte der rechtlichen Integration auch auf andere Bereiche, etwa den freien Dienstleistungs- und Personenverkehr, übertragen wurden, obwohl die funktionalen Bedürfnisse dort mitunter ganz andere sind. Die historisch-institutionalistische Perspektive ist komplexer, insgesamt aber erklärungskräftiger als rationalistische oder soziologische Ansätze. Während letztere mit starken Annahmen zur Motivation von Gerichten bzw. der Sozialisation von RichterInnen operieren, kombiniert unser Ansatz zwei weniger voraussetzungsreiche Faktoren, die eine pfadabhängige Rechtsprechung in Richtung weiterer Internationalisierung begünstigen: Zunächst bemühen sich RichterInnen um eine kohärente Rechtsprechung, so dass wachsendes Fallrecht aufgrund der Präzedenzlogik eine eigene Wirkmacht entwickelt. Darüber hinaus verstärkt das Klageverhalten privater Akteure und supranationaler Institutionen wie der Europäischen Kommission diese Tendenz, indem existierendes Fallrecht selektiv aufgegriffen und auf neue Bereiche übertragen wird. Ist die Unabhängigkeit internationaler Gerichte grundsätzlich gewollt, damit diese ihre Rolle als Streitschlichter erfüllen können, wird sie aber problematisch, wenn politische Korrekturen einmal eingeschlagener Rechtsprechungspfade nahezu ausgeschlossen sind.

Durch einen doppelten Vergleich werden die Voraussetzungen und Grenzen einer pfadabhängigen internationalen Verrechtlichung näher bestimmt und alternative Erklärungen ausgeschlossen. EU-intern wird der Entwicklung im Waren-, Dienstleistungs- und Personenverkehr die oftmals weniger beachtete Rechtsprechung zum Kapitalverkehr gegenübergestellt; EU-extern wird die Rolle des EuGH mit dem Streitschlichtungsverfahren in der WTO verglichen. Die Rechtsprechung zur Kapitalverkehrsfreiheit in der EU erzeugte lange Zeit keine mit den anderen Freiheiten vergleichbare Dynamik, was sich nicht durch das Eigeninteresse oder die Sozialisation der (identischen) EuGH-RichterInnen erklären lässt. Erst mit der Etablierung der unmittelbaren Anwendbarkeit des europäischen Vertragsrechts und zunehmenden Fallzahlen auch in diesem Bereich konnte der EuGH, positiv verstärkt von privaten KlägerInnen und der Kommission, seine Auslegung der anderen Grundfreiheiten übertragen. Auch dieser Bereich ist seitdem in die durch expansive Internationalisierung gekennzeichnete pfadabhängige Rechtsprechung des EuGH einbezogen.

Der externe Vergleich zur WTO dient auch der besseren beiderseitigen Anschlussfähigkeit von Europaforschung und IB (vgl. in ähnlicher Stoßrichtung Alter/ Helfer 2010), denn die Parallelen zwischen beiden Rechtsordnungen reichen weiter als dies in der Europaforschung meist wahrgenommen wird. Nicht nur ist der Abbau nationaler Handelsschranken das Hauptziel der WTO und es bestehen Übereinstimmungen zur EU in grundlegenden Rechtsvorschriften; vielmehr ist auch in der WTO die Gerichtsbarkeit durchaus bereit und in der Lage, eigene Handlungsspielräume kreativ zu nutzen und rechtliche Verpflichtungen von Mitgliedstaaten auszu- 
weiten. Entscheidend für die insgesamt weniger expansive Rechtsprechung in der WTO ist ein institutioneller Unterschied: Die WTO-Mitgliedstaaten kontrollieren exklusiv, mit welchen Fällen sich die Gerichtsbarkeit der WTO überhaupt beschäftigt und können so das Volumen und die eigenständige Wirkmacht des Fallrechts begrenzen.

Der weitere Text widmet sich zunächst unserem theoretischen Rahmen. Danach wird das zuvor eingeführte Argument am Beispiel des freien Waren-, Dienstleistungs- und Personenverkehrs in der EU entwickelt. Darauf folgen zwei Vergleichsstudien zum freien Kapitalverkehr in der EU sowie zur Handelsliberalisierung in der WTO, mit denen die Voraussetzungen und Grenzen des Arguments näher untersucht werden können. Abschließend werden zentrale Ergebnisse zusammengefasst und weitere Forschungsperspektiven skizziert.

\section{Internationale Gerichte, rechtliche Präzedenz und Pfadabhängigkeit}

Im Folgenden werden die Gründe für die Schaffung unabhängiger internationaler Gerichte diskutiert und anschließend aus historisch-institutionalistischer Perspektive hergeleitet, unter welchen Voraussetzungen die Rechtsprechung internationaler Gerichte pfadabhängig in Richtung weiterer Internationalisierung wirkt.

\subsection{Die Unabhängigkeit internationaler Gerichte}

Warum sollten internationale Gerichte Fallrecht schaffen, das über diejenigen Ziele und Verpflichtungen hinausgeht, die von den Staaten auch politisch unterstützt werden? Und inwiefern besitzen internationale Gerichte überhaupt die dazu nötige Unabhängigkeit?

Insbesondere zur zweiten Frage, der Unabhängigkeit internationaler Gerichte und ihren Möglichkeiten einer eigenständigen Rechtsfortbildung, besteht mittlerweile eine breit ausdifferenzierte rechts- und politikwissenschaftliche Literatur. Da sie nur über begrenzte Ressourcen verfügen und von der Implementierung richterlicher Entscheidungen durch die Mitgliedstaaten abhängig sind, gelten internationale Gerichte häufig als relativ durchsetzungsschwach (Zangl 2009b: 25). Deshalb wird die bestehende Judizialisierung auf internationaler Ebene vielfach als unzureichend angesehen, um tatsächliche Regeleinhaltung zu befördern, und eine weitere Stärkung internationaler Gerichte befürwortet (Abbott et al. 2000: 415; Zangl 2009a). Auch zeigt die Prinzipal-Agent-Literatur, dass nationale Regierungen oft nur begrenzte Entscheidungsmacht delegieren und Kontrollmechanismen gegen eine Verselbstständigung des Agenten implementieren (Tsebelis/Garrett 2001: 363).

Die Kategorisierung von Gerichten als typische Agenten wird aber auch angezweifelt (Alter 2008: 33-36). So sind internationale Verträge zumeist unvollkommen und die letztinstanzliche Auslegung von Kompetenzregeln obliegt oft Gerichten (Abbott/Snidal 2000: 433). Dabei können sie ihre Funktion zur Bewältigung 
kollektiver Handlungsprobleme nur erfüllen, wenn sie unabhängig von politischer Einflussnahme sind. In der Praxis wird dies vor allem dadurch gewährleistet, dass Urteile politisch nur schwer korrigierbar sind; Vertragsänderungen sind beispielsweise in der EU und der WTO nur einstimmig möglich. So schützt das Wirken der Politikverflechtungsfalle (Scharpf 1985) die Urteile internationaler Gerichte vor politischen Änderungen. Im Vergleich zur politisch handlungsfähigeren nationalen Ebene besitzt die Judikative auf europäischer und internationaler Ebene in dieser Hinsicht einen Machtvorteil. Deshalb plädiert Karen Alter dafür, grundsätzlich von der Unabhängigkeit internationaler Gerichte auszugehen:

$»[\ldots]$ The presumption should be in favor of IC [international court] independence rather than Principal control. Giving up the idea that states are the hidden puppet-masters of ICs allows us to instead focus on how international politics is being transformed by the existence of an alternative venue of international politics - namely international legal arenas« (Alter 2008: 55).

Oft wird der EuGH als das mächtigste internationale bzw. supranationale Gericht angesehen und sogar auf eine Stufe mit nationalen Verfassungsgerichten gestellt (Stone Sweet 2010: 2). Vorrang und unmittelbare Anwendbarkeit des Europarechts - die über die Zeit vom EuGH nicht nur für Vertragsinhalte, sondern auch für weniger hochrangige Rechtsnormen erfolgreich etabliert wurden - haben sich als die zentralen Bausteine beim Aufbau einer europäischen föderalen Ordnung erwiesen. Hinzu kommt eine Eigentümlichkeit des europäischen Rechtssystems, die nationale Gerichte zu Kooperationspartnern des EuGH macht und es den Mitgliedstaaten erschwert, dauerhaft gegen europäisches Recht zu verstoßen. Über das Vorabentscheidungsverfahren richten nationale Gerichte Fragen über die Auslegung europäischen Rechts an den EuGH - ein Verfahren, das gerade für Gerichte unterer Instanzen attraktiv ist, da ihre Urteile dann kaum von höheren nationalen Gerichten verworfen werden können. Ursprünglich als Hilfestellung bei der Auslegung europäischen Rechts geschaffen, ist hieraus im Laufe der Zeit eine europäische Normenkontrolle nationalen Rechts geworden (Alter 1998: 133-135).

Wird der EuGH aufgrund seiner Stärke in der Europaforschung häufig als einzigartig betrachtet, so schreibt die IB-Forschung auch anderen internationalen Gerichtsbarkeiten, etwa den Organen des WTO-Streitschlichtungsverfahrens, dem Europäischen Gerichtshof für Menschenrechte (EGMR) oder dem Internationalen Strafgerichtshof (IStGH) eine große Unabhängigkeit zu (Abbott et al. 2000: 406, 416). Da es sich bei der EU primär um eine Wirtschaftsgemeinschaft und weniger um eine Straf- oder Menschenrechtsgemeinschaft handelt, und außerdem die Warenverkehrsfreiheit in der WTO und der EU historisch bedingte Parallelen zeigt, ${ }^{2}$ wählen wir diesen Vergleichsfall.

Im streng juristischen Sinne verfügt die WTO nur über quasi-gerichtliche Organe, da die Berichte der sogenannten Panels und der Berufungsinstanz (Appellate

2 Besonders deutlich wird dies, vergleicht man etwa die (identischen) Rechtsvorschriften zum Verbot mengenmäßiger Ein- und Ausfuhrbeschränkungen in Art. 34 und 35 AEUV (Vertrag über die Arbeitsweise der Europäischen Union) sowie Art. XI des GATT (General Agreement on Tariffs and Trade). 
Body, $\mathrm{AB}$ ) für ihre rechtliche Bindungswirkung erst von den WTO-Mitgliedstaaten verabschiedet werden müssen. Die Berichte gelten aber als angenommen, wenn im Streitbeilegungsgremium der WTO (Dispute Settlement Body, DSB), in dem alle Mitgliedstaaten Stimmrecht haben, kein Konsens über eine Ablehnung des jeweiligen Berichtes zustande kommt. ${ }^{3}$ Hier besteht also eine nur formale Hürde, die auch noch nie relevant wurde. Während die Panels jeweils ad hoc eingesetzt werden, ist vor allem der permanente $\mathrm{AB}$ mit einem hohen Maß an Unabhängigkeit ausgestattet (Charnovitz 2002: 230). Dagegen sind die Möglichkeiten politischer Rechtsetzung durch das Festhalten der Mitgliedstaaten an anspruchsvollen Entscheidungsregeln und Quoren extrem limitiert (Ehlermann 2002: 302). Wie alle Verträge sind auch die WTO-Abkommen unvollständig und lückenhaft; gleichzeitig wird auch von den WTO-Rechtsprechungsorganen erwartet, durch Kohärenz und Verlässlichkeit der Entscheidungsfindung zu mehr Rechtssicherheit im WTO-Rechtssystem beizutragen (Stone Sweet 2004: 33). Obwohl die Präzedenzwirkung von Einzelfallentscheidungen in den WTO-Abkommen de jure nicht vorgesehen ist, wird de facto gerade aus Gründen der Rechtssicherheit eine solche Wirkung in empirischen Studien mittlerweile festgestellt (Bhala 1999; Trachtman 1999: 339). Im Lichte all dessen nimmt also gerade der $\mathrm{AB}$ eine dem EuGH nicht unähnliche Rolle bei der Bestimmung ein, ob nationale Handelsbeschränkungen (un-)zulässig sind (Scott 2004: 311).

\subsection{Die Pfadabhängigkeit der Rechtsprechung}

Auch wenn der EuGH und die Streitschlichtungsorgane der WTO beträchtliche Unabhängigkeit besitzen und nur schwer von ihren Mitgliedstaaten kontrolliert oder korrigiert werden können, heißt dies noch nicht, dass ihre Rechtsprechung notwendigerweise auf weitere Internationalisierung, auch gegen den Willen der Mitgliedstaaten, ausgerichtet sein muss. Gerade dem EuGH wird jedoch weithin eine solche Rolle als »Motor der Integration« zugeschrieben. Während die Ursachen der außergewöhnlichen Macht des EuGH, wie im vorausgehenden Abschnitt angedeutet, mittlerweile umfangreich erforscht wurden, ${ }^{4}$ erklärt dies noch nicht die spezifische pro-integrative Ausrichtung der EuGH-Rechtsprechung. Allgemeiner formuliert: Warum sollten internationale Gerichte Fallrecht schaffen, das weiter auf einem bestimmten Pfad internationaler Verrechtlichung voranschreitet, als dies von den Mitgliedstaaten politisch unterstützt wird?

3 Diese Regel negativen Konsenses ist in Art. 16.4 und 17.4 der WTO-Streitschlichtungsordnung vorgesehen.

4 Siehe die Zusammenfassung des politikwissenschaftlichen Forschungsstandes von Stone Sweet (2010). 
Bestehende Erklärungsansätze ${ }^{5}$ aus der politikwissenschaftlichen Europaforschung und der IB lassen sich meist den Lagern des rationalistischen oder soziologischen Neo-Institutionalismus (Hall/Taylor 1996: 942, 946) zuordnen. Diese werden im Folgenden kurz diskutiert, bevor anschließend ein historisch-institutionalistischer Zugang entwickelt wird.

Aus rationalistischer Sicht wird einerseits argumentiert, internationale Gerichte wie der EuGH folgten den Präferenzen der (großen) Mitgliedstaaten (Garrett 1995; Garrett et al. 1998). Dass die Rechtsprechung des EuGH weitgehend eingehalten und kaum politische Korrekturen an der Judikative vorgenommen würden, belege diese These (Garrett 1995: 172). Mittlerweile wird jedoch kaum bestritten, dass der EuGH auch oft gegen mitgliedstaatliche Interessen Recht spricht - ausbleibende Konflikte mit den Regierungen sind der kaum möglichen politischen Korrektur ebenso geschuldet wie der Tatsache, dass sich die Regeldurchsetzung auch über nationale Gerichte vollzieht (Alter 1998: 133, 136). Die folgende empirische Analyse bestätigt dies.

Andererseits wird aus rationalistischer Perspektive oft eher implizit ein richterliches Eigeninteresse an expansiver Rechtsprechung angenommen, d.h. es liegt in der »Natur« (Alter/Helfer 2010: 5) internationaler Gerichte, dass sie die internationale Verrechtlichung vorantreiben wollen. In einem der klassischen Beiträge zum EuGH als Motor der Integration heißt es dazu: »The supranational actors [...] seek unremittingly to expand the mandate of their own institutions to have a more influential say in community affairs« (Burley/Mattli 1993: 55). Eine Rückführung der weiteren Internationalisierung auf richterliches Eigeninteresse läuft jedoch Gefahr, potenziell (selbst-)beschränkende Elemente des Rechts zu unterschätzen. Zunächst sind Gerichte davon abhängig, dass sie überhaupt Fälle erreichen. Zudem können sie bestehendes Recht nicht beliebig auslegen und einmal getroffene Interpretationen nur schwer ändern, um nicht Rechtssicherheit und Kohärenz zu gefährden.

Der rationalistische Ansatz liefert somit zwei gegensätzliche Erwartungen hinsichtlich der Rechtsprechung internationaler Gerichte. Je nachdem, welches maßgebliche (Eigen-) Interesse man unterstellt, sollten internationale Gerichte die internationale Verrechtlichung also gar nicht gegen staatlichen Widerstand oder bei jeder Gelegenheit vorantreiben. Schwerer als diese Zweideutigkeit wiegt jedoch, dass beide interessenbasierten Erklärungsvarianten die Eigenlogik des Rechts nicht ernst genug nehmen (vgl. Joerges 1996). Durch bestehendes Vertrags- und Fallrecht wird die Rechtsprechung nämlich zugleich ermächtigt (gegenüber mitgliedstaatlichen Interessen) und beschränkt (gegenüber einem möglichen Eigeninteresse der RichterInnen). Den differenzierten empirischen Befund, dass internationale Gerichte die Internationalisierung des Rechts durchaus gegen staatlichen Widerstand vorantrei-

5 Als politikwissenschaftlichen Erklärungsansatz verstehen wir hierbei den Versuch, Kausalmechanismen zu identifizieren und ihre Generalisierbarkeit zu prüfen. An breitere normative, vorwiegend rechtswissenschaftliche Debatten, wie etwa zur Konstitutionalisierung und zum Rechtspluralismus (Weiler 1991; De Búrca/Scott 2003a; von Bogdandy/Bast 2010), können wir damit nur bedingt anknüpfen. 
ben, aber nicht bei jeder Gelegenheit, kann daher keine der beiden rationalistischen Varianten erklären.

Aus einer soziologischen Perspektive ist die Ausrichtung internationaler RichterInnen auf weitere Internationalisierung kein naturgegebenes Eigeninteresse, sondern in sozialer Interaktion erworben im Sinne einer Logik der Angemessenheit (March/Olsen 1998: 951). So beschreibt Antoine Vauchez (2010: 22) detailliert, wie sich in der Frühphase der europäischen Integration trotz staatlichen Widerstands ein neuer europarechtlicher Common Sense auf europäischer Ebene herausbildete und von einem Netzwerk aus AnwältInnen, RichterInnen und Angehörigen des Juristischen Dienstes der Europäischen Kommission bald aktiv gefördert wurde (vgl. auch Alter 2009: Kap. 4). In einem anderen sozialen Kontext kann der Common Sense demnach auch anders ausfallen. So liefern Alter und Laurence Helfer (2010: 20) eine im Kern soziologische Erklärung, weshalb die Rechtsprechung des Andentribunals wesentlich vorsichtiger ausfällt, obwohl es sich hier um ein institutionelles Ebenbild des EuGH handelt.

Auch aus dem soziologischen Ansatz folgt so keine eindeutige Erwartung hinsichtlich der Rechtsprechung internationaler Gerichte. Stattdessen wird die Erklärung unterschiedlich expansiver Rechtsprechung auf eine andere Ebene verlagert, den sozialen Kontext, dessen verschiedene Ausprägungen aber ihrerseits erklärungsbedürftig bleiben. ${ }^{6}$ Eine soziologische Erklärung internationaler Rechtsprechung ist aber nicht nur äußerst aufwendig, sie erscheint bei den im Folgenden diskutierten Vergleichsfällen von vorneherein nicht besonders plausibel: Die europäische Rechtsprechung zur Kapitalverkehrsfreiheit folgte jahrzehntelang nicht dem Pfad der anderen Grundfreiheiten, obwohl die RichterInnen und ihr soziales Umfeld die gleichen waren. Und obwohl sich zumindest starke Anzeichen für die Herausbildung eines ähnlichen Common Sense im Rahmen der WTO finden lassen (mitunter ist die Rede von einer »Genfer Familie«, vgl. kritisch Dillon 2008: 68), fehlt bislang eine dem EuGH vergleichbar aktivistische Rechtsprechung.

Alternativ zu rationalistischen und soziologischen Ansätzen wird im Folgenden eine historisch-institutionalistische Erklärung für die Frage entwickelt, weshalb und unter welchen Bedingungen internationale Gerichte die internationale Verrechtlichung auch gegen den Willen von Staaten vorantreiben. Die Erklärung ist weniger voraussetzungsreich als die zuvor diskutierten Alternativen, da sie kein starkes richterliches Eigeninteresse oder einen supranationalen Common Sense an-

6 Noch anspruchsvoller sind in dieser Hinsicht organisationssoziologische Erklärungen, die viele Gemeinsamkeiten mit dem soziologischen Neo-Institutionalismus aufweisen, das Verhalten von (internationalen) Organisationen aber nicht auf deren sozialen Kontext, sondern ihre innere Organisationskultur zurückführen (Dingwerth et al. 2009: 19). Einem solchen Ansatz sind angesichts der Vertraulichkeit des richterlichen Entscheidungsfindungsprozesses aber kaum überwindbare Grenzen gesetzt. Urteile des EuGH etwa liefern keinerlei Informationen über die internen Beratungen der RichterInnen, geschweige denn über das konkrete Abstimmungsverhalten oder abweichende Meinungen. Während wir organisationssoziologische Ansätze zur Erklärung unserer Fälle daher nicht weiter bedacht haben, teilen wir durchaus das Interesse an Dysfunktionalitäten, die sich aus einer verselbständigten Internationalisierung ergeben können (Barnett/Finnemore 1999; vgl. auch den nachfolgenden Abschnitt 3). 
nimmt. Zudem kann sie auch Unterschiede in der Eigendynamik internationaler Rechtsprechung erfassen. Im Zentrum dieser Perspektive steht das Konzept der Pfadabhängigkeit, dessen Sinn und Anwendbarkeit auf die Rechtsprechung internationaler Gerichte zunächst abstrakt diskutiert wird. Anschließend wird gezeigt, wie die einmal für den Warenverkehr in der EU etablierte Rechtsprechung pfadabhängig auf andere Bereiche übertragen wurde.

In einem engen Sinne meint Pfadabhängigkeit mehr als nur die »Historizität« (Beyer 2005: 12) sozialer Phänomene, sondern bezeichnet sich selbst verstärkende Prozesse: Ein Schritt in eine bestimmte Richtung zieht weitere Schritte auf demselben »Pfad« nach sich, die eine spätere Pfadumkehr immer unwahrscheinlicher machen (Mahoney 2000: 512). Es kommt zu einem lock-in, das heißt einmal gefundene Arrangements bleiben bestehen und prägen zukünftiges Handeln, selbst wenn die darin angelegten Lösungen sich als ineffizient erweisen (»potential path inefficiency«; vgl. Pierson 2000: 251-253). Welcher Pfad anfangs eingeschlagen wird, kann eher zufallsbedingt (»contingent «) sein, zum Beispiel bei einer technischen Entscheidung oder juristischen Interpretation, für die es auch Alternativen gegeben hätte. Auch muss die Pfadwahl nicht als kritischer Moment (»critical juncture«) erscheinen, sondern kann für sich genommen ein relativ unscheinbares Ereignis (»small event«) darstellen; bedeutend wird sie durch den folgenden Prozess positiver Verstärkung (»positive feedback«). Wichtig ist daher vor allem, wann und in welcher Reihenfolge (»timing and sequencing «) bestimmte Entscheidungen getroffen werden (Pierson 2000: 263).

Pfadabhängige Prozesse sind zunächst vor allem bei der Durchsetzung technischer Standards, etwa der QWERTY-Tastaturanordnung, nachgewiesen worden (David 1985: 334). Die Übertragbarkeit des Pfadabhängigkeitskonzepts auf die Politik wurde unter anderem mit den Eigenheiten kollektiven Handelns begründet (vgl. Pierson 2000: 257). Immer häufiger wird das Konzept auch zur Analyse von Rechtsprechung verwendet (Fon et al. 2005: 44, 47, 51). Als zentrales Charakteristikum des Rechts, das pfadabhängige Prozesse begünstigt, gilt dabei die Bedeutung von Präzedenzfällen für spätere Urteile:

»Legal institutions are path dependent to the extent that how litigation and judicial rulemaking proceeds, in any given area of the law at any given point in time, is fundamentally conditioned by how earlier legal disputes in that area of the law have been sequenced and resolved « (Stone Sweet 2002: 113).

»Fundamentally conditioned « ist aber nicht mit determiniert gleichzusetzen. Zwar verringert Präzedenz die Anzahl zulässiger rechtlicher Interpretationen. Auch dient eine enge Orientierung an existierendem Fallrecht den Gerichten dazu, Kohärenz und Rechtssicherheit zu gewährleisten und nicht den Verdacht willkürlicher oder politisch motivierter Entscheidungen zu erwecken (Gerhardt 2005: 909). Dennoch bleibt es Gerichten möglich, Neuinterpretationen vorzubringen, die bestehende Pfade der Rechtsprechung eingrenzen oder sogar mit ihnen brechen (McCown 2003: 979).

Die Verwendung des Konzepts der Pfadabhängigkeit ist somit nur sinnvoll, wenn damit mehr ausgesagt wird, als dass Präzedenz eine Rolle für die Rechtsprechung 
spielt. Zusätzlich bedarf es positiver Verstärker, damit sich ein Pfad verfestigt sowie institutioneller Hürden, die einen späteren Pfadwechsel verhindern. ${ }^{7}$ Positiv verstärkend wirkt vor allem das Klageverhalten privater und supranationaler Akteure, die eine bestimmte Rechtsprechung aufgreifen und ihr in neuen Bereichen zum Durchbruch verhelfen (Schmidt 2010). So schränkt Präzedenz nicht nur die Gerichte selbst in ihren zulässigen Interpretationen ein, sondern motiviert auch KlägerInnen und Beklagte, bestimmte Argumente eher vorzubringen als andere. Die besonders schwere Korrigierbarkeit internationaler Rechtsprechung wurde oben bereits angesprochen.

Wie es zum lock-in eines einmal eingeschlagenen Pfades in der Rechtsprechung kommen kann, so dass politische Ineffizienzen entstehen können, wird im nächsten Kapitel gezeigt, in dem wir uns dem Fallrecht des EuGH in den Bereichen des Waren-, Dienstleistungs-, und Personenverkehrs in der EU zuwenden. Die darauf folgenden Vergleichsfälle des Kapitalverkehrs in der EU und der Handelsliberalisierung im Rahmen der WTO dienen der Plausibilisierung des Arguments und zeigen, dass sich ohne positive Verstärker keine vergleichbare Pfadabhängigkeit entwickelt. Eine weitergehende empirische Beweisführung im Sinne des process tracing würde verlangen, im Detail nachzuzeichnen, wann etwa bei der Kapitalverkehrsfreiheit im Schrifttum, in Stellungnahmen der Generalanwälte oder in Urteilen eine parallele Argumentation zur Warenverkehrsfreiheit angedacht wurde, und welche KlägerInnen dies dann wie aufnahmen. ${ }^{8}$ Dies lässt sich im Rahmen eines Aufsatzes, der einen weiteren Bogen spannt, nicht leisten.

\section{Freier Waren-, Dienstleistungs- und Personenverkehr in der EU}

Die Vorschriften zum freien Waren-, Dienstleistungs- und Personenverkehr ${ }^{9}$ gehören zu den Grundpfeilern der Römischen Verträge und der europäischen Integration. Darauf aufbauend wurde der gemeinsame Binnenmarkt als Kernstück der Integration verwirklicht. Ähnlich wie in anderen Freihandelszonen auch, wurden die europäischen Grundfreiheiten ursprünglich als Diskriminierungsverbote formuliert

7 Diese beiden Elemente sind es auch, die unser historisch-institutionalistischer Ansatz anders betont als frühere Neofunktionalisten. Das unmittelbare »self-interest of judges, lawyers, and professors « (Burley/Mattli 1993: 60) reicht alleine nicht aus, um die rechtliche Internationalisierung eigendynamisch voranzutreiben - vielmehr braucht es private und supranationale Kläger, die das Fallrecht in oftmals unvorhergesehener Weise aufgreifen und so positiv verstärken. Darüber hinaus betonen wir die Dysfunktionalität, die die Übertragung einer kaum mehr korrigierbaren Rechtsprechung auf neue Bereiche erzeugen kann: Was rechtlich plausibel oder im Sinne größtmöglicher Kohärenz sogar notwendig erscheinen mag, muss - entgegen der funktionalistischen Annahme - nicht einhergehen mit einem »similar level of effectiveness in the broader spheres of economic, social, and political integration« (Burley/Mattli 1993: 73).

8 Für ein entsprechendes Vorgehen hinsichtlich der Anfänge des Europarechts vgl. Vauchez (2010).

9 Unter den freien Personenverkehr fallen die Arbeitnehmerfreizügigkeit und die Niederlassungsfreiheit. Der freie Kapitalverkehr wird im Anschluss an diesen Abschnitt separat behandelt. 
und interpretiert. Als Voraussetzung für grenzüberschreitenden Handel konnten Mitgliedstaaten von ausländischen Unternehmern verlangen, dass diese bei ihren Aktivitäten im Inland die für alle geltende Marktregulierung berücksichtigten; war diese Voraussetzung erfüllt, durfte nicht diskriminiert werden.

Der EuGH änderte diese Interpretation der Grundfreiheiten in den berühmten Fällen Dassonville (Rechtssache 8/74) und Cassis de Dijon (129/78) in den 1970er Jahren. Nationale Marktregulierung, so argumentierten die RichterInnen, könne wie eine quantitative Handelsbeschränkung wirken. Seither müssen Mitgliedstaaten die Marktregulierung im Herkunftsland eines Unternehmers mit berücksichtigen und gegenseitig anerkennen, wenn sie über Fragen des Marktzugangs entscheiden. Von Unternehmen darf nur die Einhaltung zusätzlicher Regeln verlangt werden, wenn dies verhältnismäßig ist. Mit seiner Auslegung der Grundfreiheiten als Verpflichtung zur gegenseitigen Anerkennung lenkte der EuGH den Integrationsprozess also auf einen ganz anderen Pfad als dies bei einem bloßen Diskriminierungsverbot der Fall gewesen wäre.

In der Frühphase der europäischen Integration konzentrierte sich der grenzüberschreitende Handel vor allem auf Waren und so wurde die Umdeutung vom Diskriminierungs- zum Beschränkungsverbot zunächst im Bereich des Warenverkehrs etabliert. In einer Gemeinschaft von neun Mitgliedstaaten mit weitgehend vergleichbarem ökonomischen Entwicklungsstand, so die Situation der 1970er-Jahre, konnten die Mitgliedstaaten von einer Äquivalenz ihrer Regulierungsniveaus und der entsprechenden Kontrollmechanismen ausgehen. Gleichzeitig konnten weitere regulative Beschränkungen mit einem übergeordneten öffentlichen Interesse wie etwa dem Umweltschutz gerechtfertigt werden.

Warum aber schwenkte auch die Interpretation der anderen Grundfreiheiten schrittweise auf diesen Pfad um? Allen voran bot eine vergleichbare Interpretation der anderen Grundfreiheiten privaten Akteuren einen größeren Handlungsspielraum auch bei anderen wirtschaftlichen Tätigkeiten. Damit bestanden große Anreize, eine Übertragung der EuGH-Rechtsprechung auf die anderen Freiheiten einzuklagen. Private Kläger haben mit dem Vorabentscheidungsverfahren über nationale Gerichte indirekten Zugang zum EuGH. Sie können so als positive Verstärkung für einen einmal eingeschlagenen Pfad der Rechtsprechung wirken, so dass diese pfadabhängig wird und sich eigendynamisch weiterentwickelt.

Ein Beispiel aus dem Bereich der Dienstleistungsfreiheit zeigt den grundlegenden Mechanismus. Eines der ersten einschlägigen Urteile, in dem die Argumentation aus Cassis auf Dienstleistungen übertragen wurde, betraf den Fall Säger (C-76/90). Hier fragte ein deutsches Gericht den EuGH, ob es mit der Dienstleistungsfreiheit vereinbar sei, dass ein im Vereinigten Königreich niedergelassener Unternehmer, der sich auf die Aufrechterhaltung von Patenten spezialisiert hatte, in Deutschland nur mit spezieller Lizenz tätig werden durfte. In dem Verfahren argumentierte nur eine Streitpartei, die englischen Patentanwälte, für eine weite Auslegung der Dienstleistungsfreiheit als Beschränkungsverbot analog der Warenverkehrsfreiheit - der deutsche Patentanwalt Säger und die Regierung des Vereinigten Königreichs bezogen sich auf die herkömmlich engere Auslegung als Diskriminie- 
rungsverbot. ${ }^{10}$ In seiner Antwort argumentierte der Gerichtshof deutlich in Analogie zur Warenverkehrsfreiheit:

»Artikel 59 EWG-Vertrag verlangt nicht nur die Beseitigung sämtlicher Diskriminierungen des Dienstleistungserbringers aufgrund seiner Staatsangehörigkeit, sondern auch die Aufhebung aller Beschränkungen - selbst wenn sie unterschiedslos für einheimische Dienstleistende wie für Dienstleistende anderer Mitgliedstaaten gelten -, wenn sie geeignet sind, die Tätigkeit des Dienstleistenden, der in einem anderen Mitgliedstaat ansässig ist und dort rechtmäßig ähnliche Dienstleistungen erbringt, zu unterbinden oder zu behindern« (EuGH 1991: Leitsatz Nr. 1).

Indem der EuGH bestehende Rechte weit auslegt oder sogar neue Rechte schafft, motiviert er andere Akteure, sich ebenfalls vor Gericht um eine Ausweitung ihres wirtschaftlichen Handlungsspielraums über das Europarecht zu bemühen. Im Mittelpunkt eines anderen grundlegenden Rechtsstreits (Gebhard, C-55/94) stand ein deutscher Rechtsanwalt, der in Mailand in einer eigenen Kanzlei praktizierte, ohne der dortigen Rechtsanwaltskammer anzugehören. Rechtlich unklar war dabei unter anderem die Abgrenzung von Dienstleistungs- und Niederlassungsfreiheit. Während Gebhard im Rahmen der Dienstleistungsfreiheit zweifellos für einen vorübergehenden Zeitraum als deutscher Rechtsanwalt in Italien praktizieren durfte, fiel seine dauerhafte Berufstätigkeit unter die Niederlassungsfreiheit, wobei er als in Italien niedergelassener Anwalt den hier geltenden Regeln unterlag. Gleichwohl, so das Urteil des Gerichtshofs, darf ein Mitgliedstaat auch im Rahmen der Niederlassungsfreiheit nur solche rechtlichen Verpflichtungen auferlegen, die vier Kriterien erfüllen:

»Sie müssen in nichtdiskriminierender Weise angewandt werden, sie müssen aus zwingenden Gründen des Allgemeininteresses gerechtfertigt sein, sie müssen geeignet sein, die Verwirklichung des mit ihnen verfolgten Zieles zu gewährleisten, und sie dürfen nicht über das hinausgehen, was zur Erreichung dieses Zieles erforderlich ist« (EuGH 1995: Leitsatz Nr. 6).

Auch die Niederlassungsfreiheit begründet somit nicht nur ein Recht auf Nichtdiskriminierung, sondern verpflichtet die Mitgliedstaaten dazu, nationale Regulierung auf ein notwendiges und verhältnismäßiges Maß zu beschränken.

Angesichts der schieren Fülle an Fallrecht wären weitere unterstützende Beispiele leicht zu nennen. Inwiefern aber lassen sich auch gegenteilige Beispiele finden, in denen der EuGH eine abweichende Rechtsprechung verfolgt oder einmal getroffene Festlegungen revidiert hat? Sie könnten Aufschluss geben, wodurch die Pfadabhängigkeit des Fallrechts ihre Grenzen findet. Eine von den restlichen Grundfreiheiten abweichende Linie verfolgte der EuGH lange Zeit hinsichtlich der Kapitalverkehrsfreiheit, die im nächsten Abschnitt behandelt wird. Das zentrale Beispiel für eine teilweise Korrektur der eigenen Rechtsprechung im Bereich der Warenverkehrsfreiheit ist das Keck-Urteil (C-267/91 und C-268/91). Darin nahm der EuGH bestimmte Verkaufsmodalitäten von der Reichweite der Warenverkehrsfreiheit aus, nachdem sich die RichterInnen zunehmend mit Fällen konfrontiert sahen, in denen

10 Vgl. Randnummern 8-12 im Schlussantrag von Generalanwalt Jacobs vom 21. Februar 1991. 
das europäische Recht als Hebel für rein nationale Reformen instrumentalisiert wurde (Rawlings 1993). Das Urteil zeigt, dass RichterInnen bestehende Interpretationspfade verlassen und neue Argumentationslinien einführen können.

$\mathrm{Zu}$ viele Pfadwechsel oder Neuinterpretationen des Pfades schaden aber der Rechtssicherheit. Aufschlussreich ist insofern der Schlussantrag von Generalanwalt Maduro in zwei Streitfällen über Anwaltsgebühren in Italien (Fälle C-94/04 und C-202/04, Rdnrn. 27-30). Zwar räumt er darin generell ein, »dass das Auftreten neuer Gesichtspunkte eine Anpassung oder sogar Revidierung (der) Rechtsprechung rechtfertigen kann« und erwähnt das Keck-Urteil als Extrembeispiel. Im konkreten Fall lehnt Maduro jedoch eine geforderte Korrektur der Rechtsprechung ab und argumentiert:

»Die Autorität, die der Gerichtshof seinen in der Vergangenheit erlassenen Urteilen zuerkennt, lässt sich als Konsequenz der Notwendigkeit begreifen, die jeder Rechtsordnung innewohnenden Werte der Stimmigkeit, Einheitlichkeit und Rechtssicherheit zu gewährleisten. Diese Werte sind in einem System der dezentralisierten Rechtsanwendung wie der Gemeinschaftsrechtsordnung von umso größerer Bedeutung « (Maduro 2006: Rdnr. 28).

Da der EuGH von der dezentralen Anwendung des Europarechts durch nationale Gerichte abhängt, orientieren sich die europäischen RichterInnen also besonders stark an Präzedenzfällen. Auch dass der EuGH immer wieder überraschend einzelne Beschränkungen der Marktfreiheiten zulässt (vgl. Voßkuhle 2010: 195), steht nicht im Widerspruch zu dieser grundsätzlichen Logik. So wird die Reichweite der Marktfreiheiten systematisch weit, die Rechtfertigungsmöglichkeiten von Beschränkungen hingegen als enge Ausnahmen interpretiert (Oliver/Roth 2004: 416).

Private KlägerInnen aber werden vor allem solche Interpretationen aufgreifen, die ihre Rechte stärken - für sie besteht somit ein klarer Anreiz, Fälle vor Gericht zu bringen, in denen sie von einer weiten Auslegung der Marktfreiheiten profitieren und nicht solche, die ihre Reichweite beschränken. Beispielsweise tendieren Klagen zum Steuerrecht vor dem EuGH inhärent dazu, das nationale Steueraufkommen zu verringern, da KlägerInnen normalerweise nur solche Fälle vor Gericht bringen, in denen sie sich eine Verringerung ihrer eigenen Steuerlast erhoffen (Genschel/ Jachtenfuchs 2011: 303). Da Urteile wie das im Fall Keck nationale Regulierungsautonomie eher schützen, sind sie kaum geeignet, große Klagewellen anzustoßen. Das Urteil zeigt auch, dass nicht jeder Präzedenzfall des EuGH eine pfadabhängige Eigendynamik begünstigt, da hierfür eine positive Verstärkung notwendig ist. Führt der EuGH aber neue Argumentationslinien ein und werden diese Pfade von Klägern verfolgt, so ist diese Unterstützung auch für den Gerichtshof von Bedeutung. Dass die individuellen Rechte aufgegriffen werden, die der Gerichtshof durch seine Rechtsprechung selbst begründet hat, stärkt auch seine eigene Legitimität (Kelemen 2006: 123).

Es sind aber nicht nur private Akteure, die als positive Verstärker wirken, wenn sie versuchen, rechtliche Interpretationen aus einem Bereich in andere Bereiche zu übertragen. Hinzu kommt, dass in vielen Fällen vor dem EuGH mehr als nur eine Grundfreiheit betroffen ist, und dass in diesen Fällen durch eine parallele Ausle- 
gung der Grundfreiheiten rechtliche Kohärenz einfacher zu erlangen ist. Im oben zitierten Beispiel des deutschen Anwalts Gebhard, der in Italien praktizierte, waren Dienstleistungs- und Niederlassungsfreiheit gleichzeitig relevant. Würden die Grundfreiheiten unterschiedlich interpretiert, könnten sich daraus widersprüchliche rechtliche Schlussfolgerungen für ein und denselben Fall ergeben (Schmidt 2010: 464).

Pfadabhängige Prozesse sind nicht nur durch positive Verstärkungen gekennzeichnet - sie lassen sich auch schwer unterbrechen und können dadurch zu ineffizienten Ergebnissen führen. Dabei muss betont werden, dass wir dem EuGH per se keinerlei Interesse an besonders strittigen Urteilen unterstellen. Vielmehr ist den RichterInnen bereits durch das europäische Primärrecht das Ziel einer »immer engeren Union« vorgegeben. Zudem muss der Gerichtshof häufig sehr spezifische Fragen entscheiden, ohne dabei, angesichts von mittlerweile 27 Mitgliedstaaten und beträchtlicher institutioneller Heterogenität, sämtliche Auswirkungen seiner Rechtsprechung abschätzen zu können. Im Gegensatz zum nationalen Verfassungskontext - so wurde bereits eingangs argumentiert - ist es den Regierungen der EU-Mitgliedstaaten aber nahezu unmöglich, die notwendige Einstimmigkeit über eine Korrektur des europäischen Vertragsrechts zu erzielen oder strittige Urteile angesichts einer integrierten Gerichtshierarchie zu missachten.

Inwiefern kann also mit Blick auf die Rechtsprechung des EuGH von Ineffizienzen gesprochen werden, wie sie die Idee eines lock-in impliziert? Rein wirtschaftlich betrachtet wird die Verwirklichung des europäischen Binnenmarktes mit Effizienzgewinnen begründet (Pelkmans 2007: 710). Während die wirtschaftliche Integration in der EU voranschreitet, bedarf die daraus erwachsende föderale politische Ordnung aber ausreichender demokratischer Legitimation. Dringt die Rechtsprechung in Bereiche vor, die ihre eigene Legitimationsgrundlage überfordern, und lässt sie sich dennoch nur schwer von den Mitgliedstaaten korrigieren, so kann dies als »politische Ineffizienz« im Sinne der Pfadabhängigkeitslogik gelten. Inwiefern sich die Rechtsprechung des EuGH noch innerhalb ihres legitimen Rahmens bewegt, ist Gegenstand einer breiten Debatte und kann auch hier nicht abschließend bewertet werden. Immerhin werden die Zweifel diesbezüglich aus unterschiedlichen politischen und wissenschaftlichen Lagern lauter (vgl. Scharpf 2009; Herzog/Gerken 2008 und nicht zuletzt die Debatte im Rahmen des Lissabon-Urteils des Bundesverfassungsgerichts). ${ }^{11}$ Als insgesamt beschränkt gilt das Legitimationspotenzial der EU insbesondere für redistributive Politiken; ihre Kernaufgabe ist die regulative Politik (Majone 1996).

Gerade die pfadabhängige Übertragung der Cassis-Logik aus dem Warenverkehr auf andere Bereiche, das zeigt schon ein schneller Durchlauf durch die verschiedenen Grundfreiheiten, wirft aber wichtige redistributive Fragen auf. Die gegenseitige Anerkennung von Dienstleistungen etwa birgt wesentlich größeres politisches Kon-

11 Das Bundesverfassungsgericht hat dort Beschwerden hinsichtlich einer Kompetenzüberschreitung des EuGH zurückgewiesen, sich aber die »Prüfung der Einhaltung des Integrationsprogramms« vorbehalten (vgl. Rdnrn. 331-340 des Urteils). 
fliktpotenzial als dies im Bereich des Warenverkehrs der Fall ist. Dies wurde in der Diskussion über die Dienstleistungsrichtlinie besonders deutlich, in deren ursprünglichem Entwurf es darum ging, das für die Warenverkehrsfreiheit etablierte Herkunftslandprinzip im Legislativprozess auf die Dienstleistungsfreiheit zu übertragen. Faktisch konnte auch die modifiziert verabschiedete Richtlinie diese Übertragung nicht verhindern, da Sekundärrecht nicht der Auslegung des Primärrechts widersprechen kann (Schmidt 2009: 860). Damit können unterschiedlich regulierte Dienstleister an einem Ort konkurrieren, so dass sich Regulierungsunterschiede der Herkunftsländer direkt in Wettbewerbsvor- und nachteile übersetzen lassen. Ähnlich ist die Situation im Rahmen der Niederlassungsfreiheit: Das Recht von Unternehmern, sich den Mitgliedstaat ihrer Niederlassung auszusuchen, bietet vielfältige Möglichkeiten, Unterschiede im Gesellschaftsrecht oder bei der Besteuerung auszunutzen (Genschel/Jachtenfuchs 2011: 302). Die expansive Auslegung der Personenfreizügigkeit in Verbindung mit der Unionsbürgerschaft hat zudem umfangreiche individuelle Anspruchsrechte geschaffen, zum Beispiel im Hinblick auf Sozialleistungen für BürgerInnen anderer Mitgliedstaaten, die die Inanspruchnahme inländischer Solidarsysteme vom Beitrag entkoppeln (Haltern 2005: 97; Scharpf 2008; Schmidt 2012).

Als Zwischenfazit lässt sich somit festhalten: Mechanismen positiver Verstärkung führen dazu, dass der einmal im Bereich des Warenverkehrs eingeschlagene Pfad auch auf die anderen Grundfreiheiten übertragen wird. Eine solche rechtliche Interpretation wäre ohne die vorherige Entwicklung im Bereich des Warenverkehrs kaum denkbar gewesen, denn sie erzeugt politisch hoch umstrittene redistributive Effekte. Diese pfadabhängige Übertragung der Rechtsprechung ist politisch nur sehr schwer korrigierbar und kann zu politischen Ineffizienzen führen, da die Legitimation redistributiver Entscheidungen auf europäischer Ebene prekär ist. Im Folgenden wenden wir uns zwei Vergleichsfällen zu, um die Erfolgsbedingungen von pfadabhängigen Prozessen genauer zu analysieren: der Kapitalverkehrsfreiheit in der EU, die lange Zeit eine Sonderrolle im System der Grundfreiheiten einnahm, und dem System der WTO, das, im internationalen Vergleich, ebenfalls relativ starke justizielle Strukturen geschaffen hat.

\section{Vergleich I: Freier Kapitalverkehr in der EU}

Neben dem freien Waren-, Dienstleistungs- und Personenverkehr ist noch eine vierte Grundfreiheit im europäischen Primärrecht verankert: die des freien Kapital- und Zahlungsverkehrs. Vor dem Vertrag von Maastricht spielte die Kapitalverkehrsfreiheit in der Rechtsprechung nur eine untergeordnete Rolle; danach lässt sich aber eine schnelle Annäherung der EuGH-Rechtsprechung an die übrigen Freiheiten beobachten. Der freie Kapitalverkehr erlaubt damit einen einzigartigen Vergleich über Zeit, um die Voraussetzungen und Grenzen einer pfadabhängigen Rechtsprechung des EuGH zu untersuchen. 
Bevor die Wirtschafts- und Währungsunion (WWU) 1990 in ihre erste Phase eintrat, war der Kapitalverkehr in Europa nur in Ansätzen liberalisiert; die vierte Grundfreiheit wurde geradezu als »Stiefkind« der anderen Freiheiten betrachtet (vg1. Barnard 2004: 461). Ohne eine engere Koordination und schrittweise Integration der Währungspolitik, so die Furcht der Mitgliedstaaten, hätte eine Lockerung der Kapitalverkehrskontrollen möglicherweise die mitgliedstaatliche Währungspolitik untergraben (Hindelang 2009: 34). Selbst die Kommission erkannte die besondere Sensibilität der Kapitalmärkte an und unterstützte Schutzmaßnahmen gegen Kapitalspekulationen in den 1970er-Jahren (Hindelang 2009: 34). In einem seiner frühesten Urteile zum freien Kapitalverkehr entschied der EuGH 1981, dass die einschlägigen europarechtlichen Bestimmungen hierzu - abweichend von den übrigen Grundfreiheiten - nicht unmittelbar anwendbar seien (Casati, C-203/80).

Durch diesen Präzedenzfall schränkten die EuGH-RichterInnen sich also selbst ein und KlägerInnen konnten den EuGH aufgrund der fehlenden Direktwirkung kaum anrufen. Für die Kapitalverkehrsfreiheit mussten die Mitgliedstaaten etwaige Integrationsschritte somit politisch entscheiden. Die wachsende Bereitschaft der mitgliedstaatlichen Regierungen, die Liberalisierung der Kapitalmärkte in Europa gegen Ende der 1980er-Jahre voranzutreiben, ist vor dem Hintergrund des WWUProjektes zu sehen (Hindelang 2009: 35-36). Am bedeutendsten war in dieser Hinsicht die 1988 vom Ministerrat beschlossene und 1990 in Kraft getretene Richtlinie 88/361/EWG. Hierin verpflichteten sich die Mitgliedstaaten, Beschränkungen des Kapitalverkehrs vollständig zu beseitigen und einigten sich auf ein breites Verständnis möglicher Kapitalbewegungen, das sie in Form einer Typologie, der sogenannten »Nomenklatur«, der Richtlinie anhängten. In einem nächsten Schritt wurde auch das europäische Vertragsrecht zum freien Kapitalverkehr durch die Reform von Maastricht zum 1. Januar 1994 grundlegend überarbeitet. Die Regeln zum freien Kapital- und Zahlungsverkehr wurden in einem Vertragskapitel zusammengefasst und im zentralen Art. 56 des EG-Vertrages verpflichteten sich die Mitgliedstaaten, weitgehend der Richtlinie folgend, alle Beschränkungen untereinander wie auch gegenüber Drittstaaten zu beseitigen (Toth 2005: 358).

Diese Neuverhandlung der Vertragsvorschriften war insofern einzigartig, als die mitgliedstaatlichen Regierungen hier die Möglichkeit hatten, aus ihren Erfahrungen mit der Rechtsprechung des EuGH zu den anderen Grundfreiheiten zu lernen (Peers 2002: 335). So führte Art. 58 I EGV eine gänzlich neue Ausnahme von der Kapitalverkehrsfreiheit ein, die eine Steuerdiskriminierung nach Wohn- bzw. Anlageort erlaubt. Diese Klausel bringt deutlich die Absicht der Mitgliedstaaten zum Ausdruck, einer möglichen juristischen Intervention in diesem sensiblen Bereich nationaler Souveränität vorzubeugen (vgl. Peers 2002: 348). Gemeinhin wurde die Klausel als Integrationsrückschritt gegenüber der Richtlinie des Rats interpretiert (Bröhmer 2007: 866; Kiemel 2003: 1775).

Bis 1994, bevor die neuen Vertragsvorschriften in Kraft traten, folgte die Integration des Kapitalverkehrs also nicht dem extensiven Internationalisierungspfad der anderen Grundfreiheiten: Die Integration kam nur in kleinen Schritten voran und wurde von den Mitgliedstaaten kontrolliert. Seit 1994 hat die Integration des Kapi- 
talverkehrs jedoch deutlich an Tempo gewonnen und die Integrationsmechanismen ähneln immer stärker denen, die im vorherigen Kapitel für die anderen Grundfreiheiten beschrieben wurden. Besonders dramatisch hat die Zahl der EuGH-Urteile zur Auslegung der Kapitalverkehrsfreiheit zugenommen - von einem Fall pro Jahr bis hin zu 19 Fällen im Jahr 2009 (siehe Abbildung 1).

Abbildung 1: EuGH-Urteile zum freien Kapitalverkehr (1978-2009)

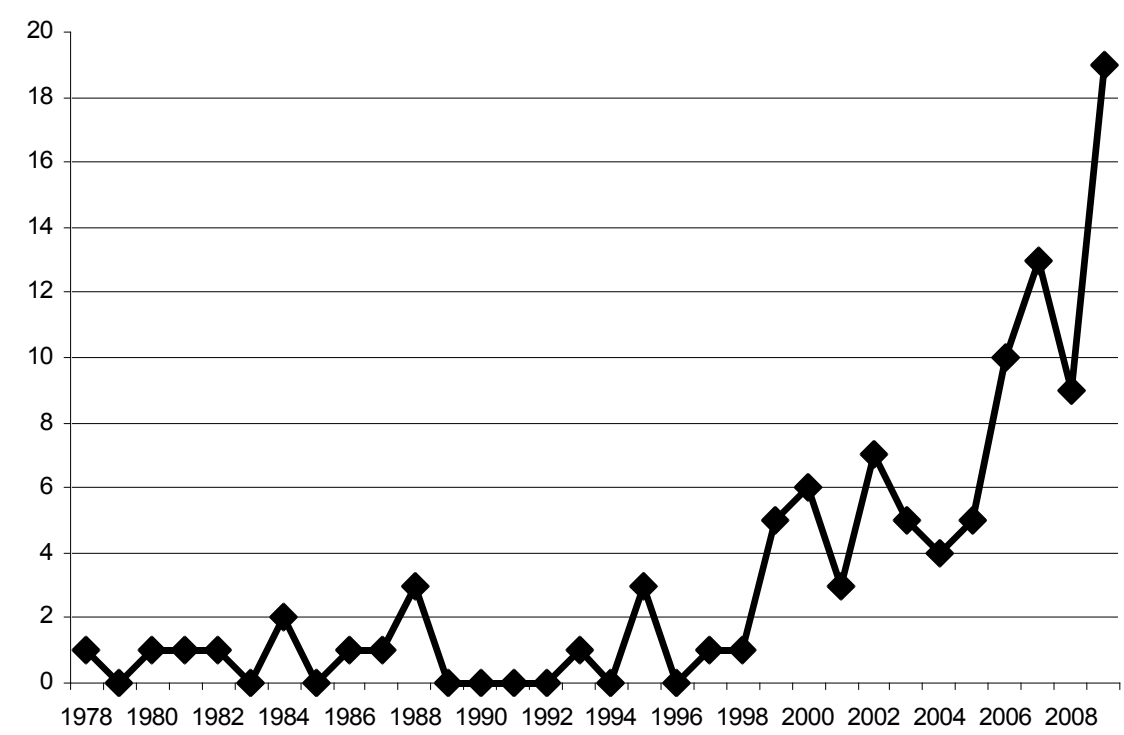

Quelle: EUR-Lex (2012)

Wie kam es zu dieser Entwicklung? Bereits 1995 befand der EuGH in einem Fall, der noch aus der Zeit vor der Maastrichter Reform stammte, dass Artikel 1 der Richtlinie 88/361 EWG hinreichend präzise und somit auf nationaler Ebene unmittelbar anwendbar sei (verbundene Rechtssachen C-358/93 und C-416/93). Da dieser Artikel inzwischen ins europäische Vertragsrecht aufgenommen worden war, hatten die Mitgliedstaaten die Auslegung der Kapitalverkehrsvorschriften »praktisch unwiderrufbar« (Kiemel 2003: 1745) an den EuGH übertragen. Die Nomenklatur im Anhang der mit der Vertragsreform formal obsoleten Richtlinie des Rats, so ein Urteil im Jahr 1999, »behält [...] den Hinweischarakter für die Definition des Begriffes des Kapitalverkehrs, den sie vor dem Inkrafttreten der letztgenannten Artikel hatte, wobei die in ihr enthaltene Aufzählung gemäß ihrer Einleitung nicht erschöpfend ist« (C-222/97, Nr. 21, unsere Hervorh.). So fällt etwa nach dem Fall 
Verkooijen (C-35/98) zusätzlich der Bezug von Dividenden einer ausländischen Gesellschaft unter die Vorschriften zum freien Kapitalverkehr.

Im gleichen Fall interpretierte der EuGH zudem den neuen Art. 58 I EGV zur Ausnahme diskriminierender Steuervorschriften derart, dass die enthaltene Ausnahmevorschrift nicht darüber hinaus geht, was ohnehin durch die frühere Richtlinie und die Rechtsprechung zu den anderen Grundfreiheiten bereits etabliert ist (C-35/98, Nr. 43). Obwohl diese Klausel ausdrücklich und nach schwierigen Verhandlungen neu ins europäische Vertragsrecht aufgenommen worden war (vgl. Hindelang 2009: 35), wurde bis heute keine Ausnahme gewährt. Ein juristischer Kommentar zum Verkoojien-Urteil resümiert kritisch:

\begin{abstract}
»While such an interpretation secures a uniform application of the Treaty freedoms as regards tax exceptions, it appears to flaunt the deliberate intentions of Treaty drafters. Thus the Court has achieved the goal of securing a coherent interpretation of the freedoms at the cost of ignoring the wording of the Treaty. As a result, any moves to adopt secondary legislation in this area will be undertaken under the shadow of the Court's jurisprudence on the primary Treaty rights, removing a great degree of discretion from national and Community legislators« (Peers 2002: 349).
\end{abstract}

Das Zitat weist bereits auf ein wesentliches Motiv für die EuGH-Rechtsprechung zur Kapitalverkehrsfreiheit nach 1994 hin, nämlich auf die systematische Einbettung der vierten Freiheit in die etablierte Rechtsdoktrin zu den anderen Marktfreiheiten. In Anlehnung an den Waren-, Dienstleistungs- und Personenverkehr interpretiert der EuGH das Beschränkungsverbot auch im Bezug auf Kapitalbewegungen sehr weit. Im ersten Urteil zu einer sogenannten "goldenen Aktie« stellte der EuGH eine Vertragsverletzung Portugals fest, da die beanstandeten portugiesischen Sonderrechte an privatisierten Unternehmen - obwohl sie In- und Ausländer gleichermaßen betrafen - den Erwerb von Unternehmensanteilen erschweren (»liable to impede «) und Anleger aus anderen Mitgliedstaaten von Investitionen abhalten könnten (»to dissuade investors«, C-367/98, Nr. 45). ${ }^{12}$ Dagegen hätte der EuGH bei einer Interpretation als Diskriminierungsverbot die Verletzung europäischer Rechte verneinen müssen. Jetzt kann grundsätzlich jede nationale Regelung, die eine Investition weniger attraktiv erscheinen lässt, unter den Vorschriften des freien Kapitalverkehrs infrage gestellt werden (Grundmann/Möslein 2003: 330).

So urteilte der EuGH im Jahr 2009 im Fall Persche etwa gegen die einhelligen Stellungnahmen Deutschlands, Frankreichs, des Vereinigten Königreichs, Irlands, Spaniens und Griechenlands, dass Spenden an gemeinnützige Einrichtungen von den Vorschriften zum freien Kapitalverkehr betroffen seien. Die Regierungen hatten sich unter anderem auf die Ausnahme zu diskriminierenden Steuervorschriften bezogen (C-318/07, Nr. 40-42). Die steuerliche Absetzbarkeit für Spenden, so jedoch der Gerichtshof, dürfe nicht auf inländische gemeinnützige Einrichtungen beschränkt werden, da sonst Spenden an ausländische Einrichtungen weniger attraktiv würden. Die oben diskutierten redistributiven Effekte, die sich aus der EuGH-

12 Eine identische Formulierung wurde im Kontext der Dienstleistungsfreiheit bereits ein Jahrzehnt zuvor im oben diskutierten Säger-Fall eingeführt (C-76/90, Nr. 12, vgl. Barnard 2004: 470). 
Rechtsprechung ergeben können, und damit ihre prekäre Legitimation werden im Bereich des Steuerrechts besonders deutlich.

Weitere Parallelen zu den anderen Grundfreiheiten fallen in der Interpretation der Kapitalverkehrsfreiheit auf: Ausnahmen der Kapitalverkehrsfreiheit werden parallel zur Rechtsprechung zum Warenverkehr seit Cassis und Dassonville (z.B. in Konle, C-302/97, siehe auch Kiemel 2003: 1736, 1773) als »zwingendes Erfordernis im Allgemeininteresse« oder durch die ausdrücklich in Art. 58 EGV genannten Gründe gewährt, wenn die geplante nationale Maßnahme einer Verhältnismäßigkeitsprüfung (siehe oben, Gebhard) standhält (Barnard 2004: 472). Ironischerweise wird diese Konvergenz nicht nur von interessierten Klägern, sondern auch von den Mitgliedstaaten positiv verstärkt, wenn sie Ausnahmen vom Beschränkungsverbot rechtfertigen. So lehnte die französische Regierung die Anwendung der Kapitalverkehrsfreiheit auf ihre goldenen Aktien ab, lieferte aber sicherheitshalber eine Begründung mit, wonach diese ein zwingendes Erfordernis im Allgemeininteresse darstellten (Lippert 2009: 344). Auf alternative Begründungsversuche, die nicht in dieses von den anderen Grundfreiheiten bekannte System gepasst hätten, ging der EuGH überhaupt nicht ein (Grundmann/Möslein 2003: 338). Besonders deutlich wurde die parallele Interpretation der Grundfreiheiten im Verfahren zum deutschen Volkswagen-Gesetz. Der Generalanwalt des EuGH war der Meinung, die Kommission habe fälschlicherweise eine Verletzung der Kapitalverkehrsfreiheit anstelle der Niederlassungsfreiheit beklagt, räumte aber letztlich ein:

»Jedenfalls lohnt es nicht, näher auf die unzutreffende rechtliche Qualifizierung der gerügten Vertragsverletzung einzugehen, da sie ohne größere Folgen bleibt; der Gerichtshof unterzieht nämlich beide Freiheiten einer ähnlichen Prüfung« (C-112/05, Nr. 60 des Schlussantrags).

In großen Teilen folgt die heutige Rechtsprechung zur Kapitalverkehrsfreiheit also dem Pfad der anderen Grundfreiheiten (Barnard 2004: 481). Ohne Direktwirkung des europäischen Vertragsrechts und angesichts einer zurückhaltenden Kommission fehlten dem EuGH lange Zeit Fälle, um sich überhaupt zur Kapitalverkehrsfreiheit äußern zu können und damit positive Verstärker, welche die Rechtsprechung hätten aufgreifen können. Im Zuge der Maastricht-Reform etablierte der EuGH dann die unmittelbare Anwendbarkeit der neu gefassten Regeln. Aufgrund dessen stiegen die Fallzahlen deutlich und die Rechtsprechung orientierte sich zunehmend an der Auslegung der anderen Grundfreiheiten - dagegen blieben Vertragsklauseln, mit denen Mitgliedstaaten einer expansiven Auslegung der Kapitalverkehrsfreiheit vorbeugen wollten, wirkungslos.

\section{Vergleich II: Handelsliberalisierung in der WTO}

Betrachtet man die Entwicklung der EU im Kontext anderer rechtlicher Integrationsprozesse, so bietet sich die WTO aufgrund vielfacher Parallelen für einen systematischen Vergleich an (Zangl 2001: 50). Primäre Aufgabe ist in beiden Fällen der Abbau nationaler Handelsschranken, wobei sich beide Organisationen dabei grund- 
sätzlich auf die gleichen wirtschaftlichen Freiheiten konzentrieren. Elementare Bestimmungen des GATT-Vertrages dienten als Blaupause für entsprechende Bestimmungen im EWG-Vertrag von 1957, und auch mit Blick auf die allgemeine Entwicklung und die Veränderung regulativer Entscheidungsmacht in der WTO lassen sich Parallelen zur EU ziehen (De Búrca/Scott 2003b: 2). EU und WTO unterscheiden sich aber mit Blick auf die zuvor diskutierten Vorbedingungen pfadabhängiger Rechtsprechung internationaler Gerichte; es fehlt ein umfangreiches Fallrecht, das von privaten Klägern selektiv aufgegriffen werden kann. Dieser institutionelle Unterschied ist maßgeblich für die abweichende Entwicklung der Rechtsprechung in der WTO und so liefert der WTO-Vergleich einen Mehrwert gegenüber dem rein EU-internen Vergleich sowie gegenüber anderen Fallstudien, die sich bewusst für minimale institutionelle Varianz entschieden haben (vgl. Alter/Helfer 2010: 3).

Im Folgenden wird gezeigt, dass eine justiziell motivierte Transformation wie in der EU in der WTO nicht bzw. nur sehr eingeschränkt stattfindet. Dabei wird durch die bestehenden Ansätze zu eigenständiger Rechtsfortbildung in der WTO deutlich, dass die unterschiedliche Entwicklung nicht auf Unterschiede im zugrunde liegenden Recht rückgeführt werden kann. Doch kommt es nicht zur Etablierung einer vergleichbar die Internationalisierung vorantreibenden Pfadabhängigkeit, da die Mitgliedstaaten, anders als in der EU, den Zugang zur Streitschlichtung kontrollieren und so positive Verstärker nur sehr begrenzt in Erscheinung treten können.

Mit Gründung der WTO richteten ihre Mitgliedstaaten eine außergewöhnlich starke internationale Gerichtsbarkeit ein. In der Zeit ihres Bestehens haben diese Streitbeilegungsorgane bereits eine wichtige Rolle beim Abbau nationaler Handelsschranken gespielt und das internationale Handelsrecht maßgeblich geprägt (Cass 2001: 72). Dass dies möglich war, ist vor allem auf das institutionelle Design der WTO sowie auf die Eigenheiten juristischer Argumentation zurückzuführen. Der obligatorische Charakter der Gerichtsbarkeit in Verbindung mit der Regel des negativen Konsenses sowie die beträchtliche Unabhängigkeit vor allem des AB machen, bei gleichzeitigem Wirken der Politikverflechtungsfalle, die relative justizielle Stärke in der WTO aus (Broude 2004: 41). Diese institutionellen Aspekte werden von Faktoren ergänzt, wie sie für viele Rechtsordnungen charakteristisch sind (lückenhafte Verträge, Präzedenzwirkung von Einzelfallentscheidungen, Kohärenz der Entscheidungsfindung), und die der WTO-Gerichtsbarkeit große Handlungsspielräume bei der Bestimmung von Zulässigkeit und Legitimität nationaler Handelsbeschränkungen einräumen.

Wie werden aber nun diese justiziellen Handlungsspielräume genutzt und lässt sich justizieller Aktivismus in der WTO beobachten (Alter/Helfer 2010: 2)? Zwei Beispiele zeigen, dass auch in der WTO Rechtsfortbildung erfolgt. In seinen Entscheidungen in US - Shrimp (WT/DS58/AB/R) und in EC - Asbestos (WT/ DS135/AB/R) entschied der AB, unaufgefordert eingesandte Amicus Curiae-Briefe (also Äußerungen Dritter, die selbst nicht Partei sind), beispielsweise von NichtRegierungsorganisationen oder Interessenverbänden, im Rahmen des WTO-Streitbeilegungsverfahrens zuzulassen, obwohl sich eine große Mehrheit von WTO-Mitgliedstaaten explizit dagegen ausgesprochen hatte: »[...] Objections ranged from 
total opposition to the argument, that the amicus curiae issue is a matter to be decided by the Members, and that the Appellate Body had transgressed the boundaries of its competences «. ${ }^{13}$ Ein zweites Beispiel ist der Bericht des Panels im Fall Australia - Automotive Leather (WT/DS126/RW), in dem das Panel befand, dass Australien zuvor rechtswidrig gezahlte Subventionen auch rückwirkend zurückzahlen sollte. Dies provozierte vehemente Proteste der Mitgliedstaaten - darunter nicht nur von Australien als unterlegener Partei, sondern auch von der Siegerin des Rechtsstreits, den USA (Ehlermann 2003: 479, Fn. 47). Das Panel hatte mit dem aus dem Instrumentarium des EuGH bekannten effet utile-Prinzip argumentiert, wonach eine Vorschrift so auszulegen ist, dass ihr Zweck möglichst umfassend erreicht wird und die Vertragsziele bestmöglich gefördert werden. Indem das Panel mit dieser teleologischen Argumentation noch über die Forderungen der Klägerin (USA) hinausging, verletzte es den Grundsatz ne ultra petita, wonach ein Gericht dem jeweiligen Kläger nicht mehr gewähren darf, als dieser beantragt hat (Bourgeois 2001: 154).

Bei diesen Beispielen und bei anderen Fällen, ${ }^{14}$ in denen sich Panels oder der AB mit Vorwürfen der Mandatsüberschreitung durch zahlreiche Mitgliedstaaten konfrontiert sahen, blieben politische Antworten der Gesamtheit der WTO-Mitgliedstaaten jedoch stets aus. Im Fall EC - Asbestos (WT/DS135/AB/R) wurde der Bericht des AB, inklusive der Entscheidung, unaufgefordert eingesandte Amicus Curiae-Briefe in Streitschlichtungsverfahren zuzulassen, angenommen und erhielt so rechtliche Bindungswirkung, da ein Konsens über seine Ablehnung nicht erreicht werden konnte. Und auch wenn die Tragweite des Panel-Berichts in Australia Automotive Leather aufgrund der außergerichtlichen Einigung von Australien und den USA limitiert bleibt - der Inhalt besteht als angenommen fort. Auch andere politische Handlungsformen wie etwa autoritative Erklärungen oder Vertragsänderungen konnten bis dato nicht genutzt werden, um die Arbeit der WTO-Rechtsprechungsorgane - fallbezogen oder fallunabhängig - zu korrigieren. Als Beispiel wäre hier ein von den USA und Chile eingebrachter Reformvorschlag ${ }^{15}$ zu nennen, der den Mitgliedstaaten stärkere Möglichkeiten geben würde, die Entscheidungsfindung des $\mathrm{AB} z u$ beeinflussen. Eine Verabschiedung dieses Vorschlags war und ist jedoch nicht in Sicht, da sich eine beträchtliche Zahl einflussreicher Mitgliedstaaten vehement gegen ihn aussprach und -spricht. Diese und weitere Vorkommnisse passen in das Bild, das auch schon für die EU gezeichnet wurde: Konzertierte politische Reaktionen unterbleiben selbst bei justiziellen Entscheidungen, die für eine große Anzahl von Mitgliedstaaten unliebsam sind. In der WTO, die mehr als 150 teilweise sehr heterogene Mitgliedstaaten zählt, entfaltet sich die Politikverflechtungsfalle (Scharpf 1985) noch viel stärker. Ereignisse wie die oben genannten, in denen die WTO-Gerichtsbarkeit autonom und über den vereinbarten Willen der

13 Ehlermann (2002: 303); vgl. auch Charnovitz (2002: 234-235) und Mavroidis (2002: 8-9).

14 Etwa India - Quantitative Restrictions (WT/DS90/AB/R), Argentina - Footwear (EC) (WT/DS121/AB/R) oder US - Lamb (WT/DS177, 178/AB/R) (vgl. Ehlermann 2002: 302-303; Alter 2008: 49).

15 Sogenanntes member control paper (TN/DS/W/52). 
Mitgliedstaaten hinaus WTO-Recht weiterentwickelt, dürften also, gerade vor dem Hintergrund der Entwicklung in der EU, durchaus zu erwarten sein.

Trotzdem ist dies nicht zu beobachten. Bei der großen Mehrheit der Panel- und AB-Berichte kommt es zu keinem Unmut der Mitgliedstaaten, denn die WTORechtsprechungsorgane nutzen ihr Potenzial zur einseitigen Rechtsentwicklung gegen den Willen der Mitgliedstaaten nur selten. Obwohl der AB sich das Recht vorbehalten hat, unaufgefordert eingesandte Amicus Curiae-Briefe in seine Entscheidungsfindung einzubeziehen, hat er bisher davon keinen Gebrauch gemacht. ${ }^{16}$ Auch die effet utile-Argumentation des Panels in Australia - Automotive Leather, die über die Forderungen der klagenden Partei hinausging, wurde in der Folge nicht mehr aufgegriffen (Vermulst/Graafsma 2005: 346). Obwohl also die Mitgliedstaaten größte Probleme haben, auf juristischem Wege getroffene Entscheidungen ex post zu korrigieren, begegnen die WTO-Rechtsprechungsorgane dem Primat politischer Entscheidungsmacht respektvoll, wo sie kritisiert werden.

Welche institutionellen Bedingungen können diese unterschiedliche Entwicklung erklären? Zunächst ist auf die Förderung politischer Konfliktlösung in der WTO zu verweisen. Ein Panel wird erst eingerichtet, wenn Konsultationen der Konfliktparteien gescheitert sind, wobei den Konfliktparteien auch im weiteren Konfliktverlauf noch Konsultations- und Mediationsmechanismen zur Verfügung stehen. ${ }^{17}$ Zahlreiche Konflikte werden so bereits auf dem Verhandlungswege entschärft oder aber zu einem späteren Zeitpunkt politisch, gewissermaßen durch die Unterbrechung des justiziellen Entscheidungsfindungsprozesses, gelöst.

Der weitere Verlauf im oben genannten Fall Australia - Automotive Leather veranschaulicht, dass politische Verhandlungen auch genutzt werden, um die innerhalb des Systems kaum blockierbaren Panel- und AB-Berichte in ihren Folgen zu begrenzen. In diesem Fall einigten sich Australien und die USA auf einen Kompromiss, der der Argumentation des Panel-Berichtes zumindest in Teilen widersprach (Arup 2003: 917). In Einzelfällen haben Parteien auch bereits angenommenen Berichten schlicht die Umsetzung verweigert (Princen 2004: 568-570). Andere Mitgliedstaaten können in solchen Fällen natürlich erneut Streitschlichtungsverfahren initiieren, was allerdings eine weitere Eskalation zur Folge hat, die eine endgültige Konfliktlösung immer schwieriger macht.

Hinzu kommt, dass die WTO der positiven Verstärkung einer integrativen Rechtsfortbildung institutionell viel weniger Spielraum bietet. Private Akteure können nur über Lobbyarbeit gegenüber nationalen Agenten der Handelspolitik (z.B. Generaldirektion Handel in der EU, United States Trade Representative und Kongressabgeordnete in den USA) auf die Einleitung von WTO-Streitbeilegungsver-

16 In diesem Zusammenhang sei beispielhaft auf den Ansatz des AB in China - Auto Parts (WT/DS339, 340, 342/AB/R) verwiesen (Abs. 11): Zwar nahmen die AB-Mitglieder den Brief an, sie stellten aber auch klar, dass »the Division hearing the appeal did not find it necessary to rely on this amicus curiae brief in rendering its decision «.

17 Bis 25. April 2012 wurden von den 436 im Rahmen der WTO vorgebrachten Streitfällen 88 Fälle einvernehmlich gelöst bzw. nach Konsultationen vom Kläger zurückgezogen; in 142 Fällen dauern die Konsultationen noch an, und zwar teilweise schon mehrere Jahre, vgl. World Trade Organization (2012). 
fahren Einfluss nehmen, da nur diese Zugang zur WTO-Gerichtsbarkeit haben. Tatsächlich kann die Kritik der Mitgliedstaaten an der AB-Entscheidung hinsichtlich der Zulassung von Amicus Curiae-Briefen als Versuch interpretiert werden, diesen Zugang weiterhin exklusiv zu halten. Die direkte Anwendbarkeit von WTO-Recht wird von nationalen Gerichten in der Regel verneint und nicht einmal die EU grundsätzlich die wohl prominenteste Verfechterin einer weiteren Verrechtlichung in der WTO - hat vor, an dieser Tatsache etwas zu ändern (Bronckers 2008: 247). Außerdem existiert in der WTO kein dem Vorabentscheidungsverfahren vergleichbares Instrument, das internationales Recht auf nationaler Ebene durchsetzen könnte (Stone Sweet 2004: 21). Die WTO-Mitgliedstaaten kontrollieren also den Zugang zum Streitbeilegungssystem und so fehlen den WTO-Streitschlichtungsorganen große Fallzahlen, mit denen eine expansive Rechtsprechung verfestigt werden könnte (Stone Sweet 2004: 30).

Auch die Möglichkeit politischer Einflussnahme durch Auswahl bzw. Wiederernennung von AB-Mitgliedern ist, wenn überhaupt, nur in Einzelfällen möglich und sicher nicht besonders effektiv. Sieht man sich die wenigen - zumeist auf Grundlage anekdotischer Evidenz dokumentierten - Beispiele solcher Einflussnahme genauer an (Steinberg 2004; Elsig/Pollack 2011), so lassen sich zwar Versuche erkennen, durch intensive nationale Auswahlverfahren die Bedrohung unliebsamer Entscheidungen zu reduzieren. Allerdings hat ein solches ausführliches nationales Prüfen amerikanischer KandidatInnen offensichtlich nicht dazu geführt, dass die letztlich nominierte und in der WTO gewählte Kandidatin zur Zufriedenheit der USA gearbeitet hätte: Berichten zufolge wird besagtem AB-Mitglied jedenfalls eine zweite Amtszeit verwehrt (Hufbauer 2011). Systematische Versuche politischer Einflussnahme durch die Auswahl der RichterInnen und mit dem Ziel der Schonung nationaler Souveränität sind überhaupt nur für die USA behauptet worden. Die Erfolgsaussichten solcher Versuche erscheinen in Anbetracht des Kollegialitätsprinzips $^{18}$ im AB, der jeweils randomisierten Fallzuweisung der Mitglieder ${ }^{19}$ und der bereits angesprochenen generellen Schwierigkeit politischer Einflussnahme als bestenfalls gering.

Zusammenfassend lässt sich sagen, dass in der Rechtsprechung des AB zwar Ansätze von Rechtsfortbildung vorhanden sind, die einen Pfad expansiver Rechtsprechung vergleichbar der Entwicklung in der EU begründen könnten. Allerdings wird die dafür notwendige positive Verstärkung unterbunden, da die Mitgliedstaaten den Zugang zur richterlichen Streitbeilegung kontrollieren und auch jederzeit auf eine politische Konfliktlösung zurückgreifen können, so dass private Akteure nicht zur Entwicklung eines umfangreichen Fallrechts beitragen können.

18 Working Procedures for Appellate Review (WT/AB/WP/1), Art. 4.

19 Working Procedures for Appellate Review (WT/AB/WP/1), Art. 6 (2). 


\section{Schlussfolgerungen}

Der vorliegende Text nimmt die wachsende Bedeutung internationaler Gerichte im internationalen Mehrebenensystem zum Ausgangspunkt. Wo die Möglichkeiten politischer Intervention in justizielle Entscheidungsabläufe stark begrenzt oder gar nicht vorhanden sind, sind internationale Gerichte mehr als bloße Agenten ihrer Prinzipale (Alter 2008: 55). Das bedeutet aber nicht, dass sie notwendigerweise eine aktivistische oder expansive Rechtsprechung verfolgen. In diesem Kontext liefert dieser Beitrag eine historisch-institutionalistische Erklärung für die Frage, weshalb und unter welchen Bedingungen internationale Gerichte den Prozess internationaler Verrechtlichung auch gegen staatlichen Widerstand vorantreiben. Das Erfordernis von Kohärenz angesichts wachsenden Fallrechts und die positive Verstärkung durch Kläger, so zeigt sich, begünstigen eine pfadabhängige Rechtsprechung zugunsten weiterer Internationalisierung.

Insbesondere der WTO-Vergleich verdeutlicht, wie voraussetzungsvoll eine eigendynamische Integration durch Recht ist. Da private Akteure ohne eigenständigen Zugang zum Streitschlichtungssystem der WTO nicht als positive Verstärker wirken können, hat sich die WTO-Rechtsprechung weit weniger expansiv entwickelt, obwohl Ansätze zu einem solchen Pfad durch die Rechtsprechung geschaffen wurden. Der Vergleich dient damit als Warnung, nicht vorschnell vom besonderen Fall des EuGH auf andere internationale Gerichte zu verallgemeinern. Die EU-Forschung kann zudem auch gewinnbringend innerhalb ihres eigenen Untersuchungsfeldes vergleichen. So zeigt die Entwicklung der Kapitalverkehrsfreiheit, wie der EuGH eine einmal gefundene Interpretation seiner bereits etablierten Jurisprudenz auf einen anderen Bereich übertragen hat. Dies geschah allerdings erst, nachdem die Mitgliedstaaten auch die Kapitalverkehrsfreiheit für unmittelbar anwendbar erklärten, so dass der EuGH mit wachsenden Fallzahlen und der zusätzlichen Herausforderung konfrontiert war, eine kohärente Rechtsprechung zu gewährleisten. Kommen zu der inhärenten Beständigkeit der juristischen Präzedenzlogik positive Verstärker hinzu, ist es wahrscheinlich, dass internationale Gerichte auf dem einmal eingeschlagenen Pfad internationaler Verrechtlichung weiter voranschreiten. Ein Fall wie Keck zeigt besonders deutlich, dass RichterInnen einen expansiven Rechtsprechungspfad auch begrenzen können. Nur dort, wo internationales Recht privaten Akteuren zusätzliche Handlungsspielräume eröffnet, sind diese aber motiviert, durch Klagen weitere Fälle an die Gerichte heranzutragen und damit diese Rechtsprechung positiv zu verstärken.

Für die IB kann das Beispiel der pfadabhängigen Rechtsprechung des EuGH als Mahnung dienen, den mit der internationalen Verrechtlichung einhergehenden Trend von (macht-)politischer zu justizieller Entscheidungsfindung stärker zu problematisieren. Teilweise werden sehr hohe Erwartungen an die internationale Verrechtlichung und die Rolle internationaler Gerichte geknüpft, von einer friedlicheren Streitbeilegung bis hin zu einer Demokratisierung globaler Politik (vgl. Zangl 2009b: 21-24). Das europäische Beispiel verdeutlicht dagegen die kritischen Aspekte einer Gewaltenverschiebung von der Legislative zur Judikative im Zuge in- 
ternationaler Verrechtlichung. Während Gerichte bereits auf nationaler Ebene eine wichtige Rolle bei der Entscheidung kontroverser und oft fundamentaler Fragen spielen, wird ihnen diese Rolle auf internationaler Ebene geradezu aufgedrängt. Werden internationale Gerichte angerufen, müssen sie zu einer Entscheidung kommen - unabhängig davon, wie unklar oder umstritten die zugrunde liegenden Regeln sind. Im Gegensatz zur nationalen Ebene steht der Judikative aber auf internationaler Ebene kein vergleichbar effizienter politischer Entscheidungsprozess zur Seite. So schaffen internationale Gerichte in großem Maße Fallrecht, das keiner politischen Beeinflussung unterworfen ist. Unter diesen Bedingungen muss die Legitimität gerichtlicher Entscheidungen besonders hohen Ansprüchen genügen, die sie aber alleine kaum erzeugen kann (Maduro 2007: 824).

\section{Literatur}

Abbott, Kenneth W./Keohane, Robert O./Moravcsik, Andrew/Slaughter, Anne-Marie/Snidal, Duncan 2000: The Concept of Legalization, in: International Organization 54: 3, 401-419.

Abbott, Kenneth W./Snidal, Duncan 2000: Hard and Soft Law in International Governance, in: International Organization 54: 3, 421-456.

Alter, Karen J. 1998: Who are the »Masters of the Treaty«? European Governments and the European Court of Justice, in: International Organization 52: 1, 121-147.

Alter, Karen J. 2008. Agents or Trustees? International Courts in their Political Context, in: European Journal of International Relations 14: 1, 33-63.

Alter, Karen J. 2009. The European Court's Political Power: Selected Essays, New York, NY.

Alter, Karen J./Helfer, Laurence 2010: Nature or Nurture? Judicial Law Making in the European Court of Justice and the Andean Tribunal of Justice, in: International Organization 64: 4, 563-592.

Arup, Christopher 2003: The State of Play of Dispute Settlement »Law« at the World Trade Organization, in: Journal of World Trade 37: 5, 897-920.

Barnard, Catherine 2004: The Substantive Law of the EU: The Four Freedoms, Oxford.

Barnett, Michael N./Finnemore, Martha 1999: The Politics, Power, and Pathologies of International Organizations, in: International Organization 53: 4, 699-732.

Beyer, Jürgen 2005: Pfadabhängigkeit ist nicht gleich Pfadabhängigkeit! Wider den impliziten Konservatismus eines gängigen Konzepts, in: Zeitschrift für Soziologie 34: 1, 5-21.

Bhala, Raj 1999: The Precedent Setters: De Facto Stare Decisis in WTO Adjudication (Part Two of a Trilogy), in: Journal of Transnational Law and Policy 9: 1, 1-151.

Bourgeois, Jacques H. J. 2001: Some Reflections on the WTO Dispute Settlement System from a Practitioner's Perspective, in: Journal of International Economic Law 4: 1, 145-154.

Bröhmer, Jürgen 2007: Der Kapital- und Zahlungsverkehr, in: Callies, Christian/Ruffert, Matthias (Hrsg.): EUV/EGV. Das Verfassungsrecht der Europäischen Union mit Europäischer Grundrechtecharta. Kommentar, München, 863-902.

Bronckers, Marco C. E. J. 2008: Private Appeals to WTO Law: An Update, in: Journal of World Trade 42: 2, 245-260.

Broude, Tomer 2004: International Governance in the WTO: Judicial Boundaries and Political Capitulation, London.

Burley, Anne-Marie/Mattli, Walter 1993: Europe Before the Court: A Political Theory of Legal Integration, in: International Organization 47: 1, 41-76. 
Cass, Deborah Z. 2001: The »Constitutionalization« of International Trade Law: Judicial Norm Generation as the Engine of Constitutional Development in International Trade, in: European Journal of International Law 12: 1, 39-75.

Charnovitz, Steve 2002: Judicial Independence in the World Trade Organization, in: Boisson de Chazournes, Laurence/Romano, Cesare/Mackenzie, Ruth (Hrsg.): International Organizations and International Dispute Settlement: Trends and Prospects, New York, NY, 219-240.

David, Paul A. 1985: Clio and the Economics of QWERTY, in: American Economic Review 75: 2, 332-337.

De Búrca, Grainne/Scott, Joanne (Hrsg.) 2003a: The EU and the WTO: Legal and Constitutional Issues, Oxford.

De Búrca, Grainne/Scott, Joanne 2003b: The Impact of the WTO on EU Decision-Making, in: De Búrca/Scott 2003a, 1-30.

Dillon, Sara A. 2008: Opportunism and the WTO: Corporations, Academics and »Member States«, in: Picker, Colin B./Bunn, Isabella D./Arner, Douglas W. (Hrsg.): International Economic Law: The State and Future of the Discipline, Portland, OR, 53-72.

Dingwerth, Klaus/Kerwer, Dieter/Nölke, Andreas 2009: Einleitung. Internationale Politik und Organisationen, in: Dingwerth, Klaus/Kerwer, Dieter/Nölke, Andreas (Hrsg.): Die Organisierte Welt. Internationale Beziehungen und Organisationsforschung, Baden-Baden, 13-37.

Ehlermann, Claus-Dieter 2002: Tensions between the Dispute Settlement Process and the Diplomatic and Treaty-Making Activities of the WTO, in: World Trade Review 1: 3, 301-308.

Ehlermann, Claus-Dieter 2003: Reflections on the Appellate Body of the WTO, in: Journal of International Economic Law 6: 3, 695-708.

Elsig, Manfred/Pollack, Mark A. 2011: Agents, Trustees, and International Courts: The Politics of Judicial Appointment at the World Trade Organization, in: http://ssrn.com/ abstract=1861443; 05.09.2011.

EuGH 1991: Urteil des Gerichtshofes vom 25. Juli 1991. Manfred Säger gegen Dennemeyer \& Co Ltd, Rechtssache C-76/90, Luxemburg.

EuGH 1995: Urteil des Gerichtshofes vom 30. November 1995. Reinhard Gebhard gegen Consiglio dell'ordine degli avvocati e procuratori di Milano, Rechtssache C-55/94, Luxemburg.

EUR-Lex 2012: Einfache Suche nach Urteilen des Gerichtshofs, Sachgebiet »Freier Kapitalverkehr «, in: http://eur-lex.europa.eu/RECH jurisprudence.do; 23.03.2012.

Fon, Vincy/Parisi, Francesco/Depoorter, Ben 2005: Litigation, Judicial Path-Dependence, and Legal Change, in: European Journal of Law and Economics 20: 1, 43-56.

Garrett, Geoffrey 1995: The Politics of Legal Integration in the European Union, in: International Organization 49: 1, 171-181.

Garrett, Geoffrey/Kelemen, R. Daniel/Schulz, Heiner 1998: The European Court of Justice, National Governance, and Legal Integration in the European Union, in: International Organization 52: 1, 149-176.

Genschel, Philipp/Jachtenfuchs, Markus 2011: How the European Union Constrains the State: Multilevel Governance of Taxation, in: European Journal of Political Research 50: 1, 293-314.

Gerhardt, Michael J. 2005: The Limited Path Dependency of Precedent, in: Journal of Constitutional Law 7: 4, 903-1000.

Grundmann, Stefan/Möslein, Florian 2003: Die goldene Aktie - Staatskontrollrechte in Europarecht und wirtschaftspolitischer Bewertung, in: Zeitschrift für Unternehmens- und Gesellschaftsrecht 32: 3, 317-366.

Hall, Peter A./Taylor, Rosemary C. R. 1996: Political Science and the Three New Institutionalisms, in: Political Studies 44: 4, 936-957.

Haltern, Ulrich 2005: Das Janusgesicht der Unionsbürgerschaft, in: Swiss Political Science Review 11: 1, 87-117. 
Herzog, Roman/Gerken, Lüder 2008: Stoppt den Europäischen Gerichtshof, in: Frankfurter Allgemeine Zeitung, 08.09.2008, 8.

Hindelang, Steffen 2009: The Free Movement of Capital and Foreign Direct Investment: The Scope of Protection in EU Law, Oxford.

Hufbauer, Gary C. 2011: WTO Judicial Appointments: Bad Omen for the Trading System, in: http://www.piie.com/realtime/?p=2209; 05.09.2011.

Joerges, Christian 1996: Taking the Law Seriously: On Political Science and the Role of Law in the Process of European Integration, in: European Law Journal 2: 2, 105-35.

Kelemen, R. Daniel 2006: Suing for Europe: Adversarial Legalism and European Governance, in: Comparative Political Studies 39: 1, 101-127.

Kiemel, Wolfgang 2003: Freier Kapital- und Zahlungsverkehr, in: von der Groeben, Hans/ Schwarze, Jürgen (Hrsg.): Kommentar zum Vertrag über die Europäische Union und Vertrag zur Gründung der Europäischen Gemeinschaft, Baden-Baden, 1706-1794.

Lippert, André 2009: Der EuGH und die Goldenen Aktien - zugleich ein Beitrag zur Dogmatik der Kapitalverkehrsfreiheit, in: JURA 31: 5, 342-348.

List, Martin/Zangl, Bernhard 2003: Verrechtlichung, in: Hellmann, Gunther/Wolf, Klaus D./ Zürn, Michael (Hrsg.): Die neuen internationalen Beziehungen. Forschungsstand und Perspektiven in Deutschland, Baden-Baden, 361-399.

Maduro, Miguel P. 2006: Schlussanträge des Generalanwalts in der Rechtssache C-94/04 Federico Cipolla gegen Rosaria Portolese in Fazari und Rechtssache C-202/04 Stefano Macrino,Claudia Capodarte gegen Roberto Meloni, Luxemburg.

Maduro, Miguel P. 2007: So Close and Yet So Far: The Paradoxes of Mutual Recognition, in: Journal of European Public Policy 14: 5, 814-825.

Mahoney, James 2000: Path Dependence in Historical Sociology, in: Theory and Society 29: 4, $507-548$.

Majone, Giandomenico (Hrsg.) 1996: Regulating Europe, New York, NY.

March, James G./Olsen, Johan P. 1998: The Institutional Dynamics of International Political Orders, in: International Organization 52: 4, 943-969.

Mavroidis, Petros C. 2002: Amicus Curiae Briefs before the WTO: Much Ado about Nothing, in: von Bogdandy, Armin/Mavroidis, Petros C./Mény, Yves (Hrsg.): European Integration and International Co-ordination: Studies in Transnational Economic Law in Honour of Claus-Dieter Ehlermann, Dordrecht, 317-329.

McCown, Margaret 2003: The European Parliament Before the Bench: ECJ Precedent and EP Litigation Strategies, in: Journal of European Public Policy 10: 6, 974-995.

Oliver, Peter/Roth, Wulf-Henning 2004: The Internal Market and the Four Freedoms, in: Common Market Law Review 41: 2, 407-441.

Peers, Steve 2002: Free Movement of Capital: Learning Lessons or Slipping on Split Milk?, in: Barnard, Catherine/Scott, Joanne (Hrsg.): The Law of the Single European Market: Unpacking the Premises, Oxford, 333-349.

Pelkmans, Jacques 2007: Mutual Recognition in Goods: On Promises and Disillusions, in: Journal of European Public Policy 14: 5, 699-716.

Pierson, Paul 2000: Increasing Returns, Path Dependence, and the Study of Politics, in: American Political Science Review 94: 2, 251-267.

Princen, Sebastiaan 2004: EC Compliance with WTO Law: The Interplay of Law and Politics, in: European Journal of International Law 15: 3, 555-574.

Rawlings, Richard 1993: The Eurolaw Game: Some Deductions from a Saga, in: Journal of Law and Society 20: 3, 309-340.

Scharpf, Fritz W. 1985: Die Politikverflechtungs-Falle. Europäische Integration und deutscher Föderalismus im Vergleich, in: Politische Vierteljahresschrift 26: 4, 323-356.

Scharpf, Fritz W. 2008: Individualrechte gegen nationale Solidarität. Eine Nachbetrachtung, in: Höpner, Martin/Schäfer, Armin (Hrsg.): Die Politische Ökonomie der europäischen Integration, Frankfurt a. M., 89-100. 
Scharpf, Fritz W. 2009: Legitimität im europäischen Mehrebenensystem, in: Leviathan 37: 2, 244-280.

Schmidt, Susanne K. 2009: When Efficiency Results in Redistribution: The Conflict over the Single Services Market, in: West European Politics 32: 4, 847-865.

Schmidt, Susanne K. 2010: Gefangen im »lock in«? Zur Pfadabhängigkeit der Rechtsprechung des Europäischen Gerichtshofs, in: Der Moderne Staat 3: 2, 455-473.

Schmidt, Susanne K. 2012: Who Cares about Nationality? The Path-Dependent Case Law of the ECJ from Goods to Citizens, in: Journal of European Public Policy 19: 1, i.E.

Scott, Joanne 2004: International Trade and Environmental Governance: Relating Rules (and Standards) in the EU and the WTO, in: European Journal of International Law 15: 2, 307-354.

Steinberg, Richard H. 2004: Judicial Lawmaking at the WTO: Discursive, Constitutional, and Political Constraints, in: The American Journal of International Law 98: 2, 247-275.

Stone Sweet, Alec 2002: Path Dependence, Precedent, and Judicial Power, in: Shapiro, Martin/ Stone Sweet, Alec (Hrsg.): On Law, Politics, and Judicialization, New York, NY, 112-135.

Stone Sweet, Alec 2004: The Judicial Construction of Europe, New York, NY.

Stone Sweet, Alec 2010: The European Court of Justice and the Judicialization of EU Governance, in: Living Reviews in European Governance 5: 2, 1-50.

Toth, Akos G. 2005: The Oxford Encyclopaedia of European Community Law: Volume II The Law of the Single Market, New York, NY.

Trachtman, Joel P. 1999: The Domain of WTO Dispute Resolution, in: Harvard International Law Journal 40: 2, 333-377.

Tsebelis, George/Garrett, Geoffrey 2001: The Institutional Foundations of Intergovernementalism and Supranationalism in the European Union, in: International Organization 55: 2, 357-390.

Vauchez, Antoine 2010: The Transnational Politics of Judicialization: Van Gend en Loos and the Making of EU Polity, in: European Law Journal 16: 1, 1-28.

Vermulst, Edwin A./Graafsma, Folkert 2005: WTO Dispute Settlement: Anti-Dumping, Countervailing Duties and Safeguards, London.

von Bogdandy, Armin/Bast, Jürgen (Hrsg.) 2010: Principles of European Constitutional Law. Modern Studies in European Law, Oxford.

Voßkuhle, Andreas 2010: Multilevel Cooperation of the European Constitutional Courts. Der Europäische Verfassungsgerichtsverbund, in: European Constitutional Law Review 6: 2, 175-198.

Weiler, Joseph H. H. 1991: The Transformation of Europe, in: The Yale Law Journal 100: 1, 2402-2483.

World Trade Organization 2012: Current Status of Disputes, in: http://www.wto.org/english/ tratop_e/dispu_e/dispu_current_status_e.htm; 25.04.2012.

Zangl, Bernhard 2001: Bringing Courts Back In. Normdurchsetzung im GATT, in der WTO und der EG, in: Swiss Political Science Review 7: 2, 49-80.

Zangl, Bernhard (Hrsg.) 2009a: Auf dem Weg zu internationaler Rechtsherrschaft? Streitbeilegung zwischen Politik und Recht. Frankfurt a. M.

Zangl, Bernhard 2009b: Judizialisierung als Bestandteil internationaler Rechtsherrschaft. Theoretische Debatten, in: Zangl 2009a, 11-35.

Zürn, Michael 2005: Introduction: Law and Compliance at Different Levels, in: Zürn, Michael/Joerges, Christian (Hrsg.): Law and Governance in Postnational Europe: Compliance beyond the Nation-State, Cambridge, 1-39. 